\title{
SMALL STRAIN OSCILLATORY SQUEEZE FILM FLOW OF SIMPLE FLUIDS
}

\author{
N. PHAN-THIEN
}

(Received 25 September 1979)

(Revised 5 November 1979)

\begin{abstract}
The oscillatory squeeze film problem is solved for the simple fluid in the sense of Noll. It is shown that dynamic properties of polymeric liquids can be measured on the plastometer in the oscillatory mode. This should be useful to food and other technologists who have to deal with awkward, highly viscous materials.
\end{abstract}

\section{Introduction}

The flow behaviour of a thin film of liquid squeezed between two circular flat plates is of continual interest to many groups of workers. Rheologists are concerned with this problem since it is the basic geometry of the popular plastometer method of determining rheological behaviour of highly viscous materials.

For the past two decades or so, there has been a controversy concerning the possible effects of lubricant viscoelasticity. The analyses of Tanner [6] and Kramer [3] predict that the elasticity of a liquid decreases its load-bearing capacity. On the other hand, Parlato [5] shows that viscoelastic liquids are better lubricants than Newtonian liquids of the same viscosity, by appealing to the "extensional primary field" approximation of Metzner [4]. This approximation may be criticized for a number of theoretical reasons [7]. The problem is mathematically intractable even for Newtonian fluids for a simple reason that the flow field is a. mixture of shear and elongation flows. Recent progress has been summarized in Brindley et al: : [1].

In this communication, we do not attempt to dwell with the complicated steady squeeze film problem mentioned above; instead, a mathematically tráctable aspect of the squeeze film flow is presented: the oscillatory squeeze film of an 
incompressible simple fluid in the sense of Noll (see Huilgol [2]). It is believed that this geometry is fairly simple to make experiments with, and consequently is worth exploring theoretically.

\section{Problem formulation}

We consider the basic squeeze film geometry which consists of two circular flat plates of radius $a$ oscillating along the $z$ axis, see Fig. 1. Half of the gap width is denoted by $h$ :

$$
h(t)=h_{0}\left(1+\varepsilon e^{j \omega t}\right), \quad j^{2}=-1,
$$

where $\varepsilon$ is a small parameter such that terms of order $O\left(\varepsilon^{2}\right)$ and higher can be neglected; a reference gap width is $h_{0}$. In normal commercial plastometers, the ratio $h_{0} / a$ is kept small.
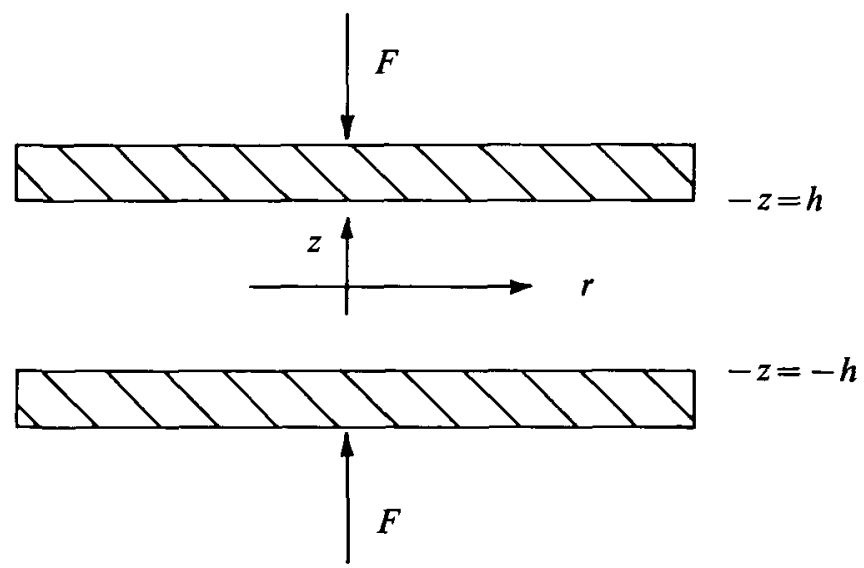

Fig. 1. Squeeze film geometry.

The total stress in an incompressible simple fluid can be shown to be (see [2])

$$
\mathbf{T}=-P \mathbf{I}+\underset{s=0}{\infty} \mathscr{H}\left(\mathbf{B}_{r}(s)\right)
$$

where $P$ is the indeterminate hydrostatic pressure and $\mathscr{H}_{s=0}^{\infty}\left(\mathrm{B}_{\ell}(s)\right)$ is a functional of a strain history $B_{t}(s)=C_{t}(s)-I$, where $C_{t}(s)$ is the Cauchy-Green tensor.

In this particular problem, the strain field is of the order $O(\varepsilon)$, the velocity field is of the order $O(\omega \varepsilon)$. By expanding (2) about a rest history (see [2]), the most general constitutive relation can be expressed as

$$
\mathbf{T}=-P \mathbf{I}+2 \int_{0}^{\infty} G(s) \mathbf{D}(t-s) d s+\underset{s=0}{\mathbf{R}}\left(\mathbf{B}_{t}(s)\right),
$$


where the error term $\mathbf{R}_{s=0}^{\infty}\left(\mathbf{B}_{t}(s)\right)$ is of the order $O\left(\varepsilon^{2}\right)$ and will be neglected. Equation (3) then becomes the general linear viscoelastic constitutive relation, with $G(s)$ being the relaxation modulus of the liquid and $\mathbf{D}$ the symmetric part of the velocity gradient tensor:

$$
D=\frac{1}{2}\left(\nabla \mathbf{u}+\nabla \mathbf{u}^{\mathbf{T}}\right) \text {. }
$$

Similarly, up to an error term of order $O\left(\varepsilon^{2}\right)$, the equation of motion reduces to

$$
\nabla \cdot \tau=\nabla P+\rho \frac{\partial \mathbf{u}}{\partial t}
$$

where $\rho$ is the fluid density, $\mathbf{u}$, the velocity, and $\tau$, the extra stress:

$$
\tau=2 \int_{0}^{\infty} G(s) \mathrm{D}(t-s) d s
$$

From (5) and (6), and using the incompressibility constraint $\nabla \cdot \mathbf{u}=0$, it is easy to see that

$$
\int_{0}^{\infty} G(s) \nabla^{4} \mathbf{u}(t-s) d s=\rho \frac{\partial}{\partial t} \nabla^{2} \mathbf{u} .
$$

To complete the problem the following boundary conditions are applied at $z=h$ :

$$
u=0 \text { and } v=h=j \omega \varepsilon h_{0} e^{j \omega t},
$$

where $u$ and $v$ are the radial and vertical velocity of the fluid, respectively. Note also that symmetry requires that $u$ be an even and $v$ be an odd function of $z$.

\section{Method of solution}

To satisfy continuity identically, it is convenient to work with the stream function $\psi$ defined by

$$
u=-\frac{\partial \psi}{\partial z}, \quad v=\frac{1}{r} \frac{\partial(r \psi)}{\partial r}
$$

Using (9) in (7) one can show that the stream function satisfies the following equation,

$$
\rho \frac{\partial}{\partial t} D^{2} \psi=\int_{0}^{\infty} G(s) D^{4} \psi(t-s) d s
$$


where $D^{2}$ is a differential operator defined by

$$
D^{2} \equiv \frac{\partial^{2}}{\partial z^{2}}+\frac{\partial}{\partial r} \frac{1}{r} \frac{\partial}{\partial r} r
$$

The general solution of $(10)$ is

$$
\psi=\psi_{1}+\psi_{2},
$$

where $\psi_{1}$ and $\psi_{2}$ satisfy the following:

$$
D^{2} \psi_{1}=0 \text { and } \rho \frac{\partial \psi_{2}}{\partial t}=\int_{0}^{\infty} G(s) D^{2} \psi_{2}(t-s) d s .
$$

The form of the boundary conditions suggests that

$$
\psi_{1}=r e^{j \omega t} \Omega_{1} \text { and } \psi_{2}=r e^{j \omega t} \Omega_{2},
$$

where $\Omega_{1}, \Omega_{2}$ are functions of $z$ and satisfy

$$
\frac{d^{2} \Omega_{1}}{d z^{2}}=0 \text { and } \frac{d^{2} \Omega_{2}}{d z^{2}}-\frac{j \omega \rho}{\eta^{*}} \Omega_{2}=0
$$

where $\eta^{*}(\omega)$ is the complex viscosity of the liquid defined by

$$
\eta^{*}(\omega)=\int_{0}^{\infty} G(s) e^{-j \omega s} d s
$$

Together with the symmetry requirement, it can be seen that

$$
\psi=r e^{j \omega t}(A z+B \sinh \alpha z),
$$

where $A$ and $B$ are integration constants and $\alpha^{2}=j \omega \rho / \eta^{*}$.

The boundary conditions (8) can be used to evaluate $A$ and $B$, from which the velocity field can be expressed as

$$
u=j \omega \varepsilon h_{0} \alpha r e^{j \omega t} \frac{\cosh \alpha h-\cosh \alpha z}{2(\sinh \alpha h-\alpha h \cosh \alpha h)}
$$

and

$$
v=2 j \omega \varepsilon h_{0} e^{j \omega t} \frac{\sinh \alpha z-\alpha z \cosh \alpha h}{2(\sinh \alpha h-\alpha h \cosh \alpha h)} .
$$

Up to now, we have not made use of the fact that $h / a \ll 1$, and (16) and (17) are the general solution of any viscoelastic fluid to the oscillatory squeeze film problem including inertial effects. 
One can now expand (16) and (17) with $h / a$ small and obtain, up to an error of order $O\left(h^{4} / a^{4}\right)$

$$
\begin{aligned}
u & =-\frac{3}{4} j \omega \varepsilon h_{0} r e^{j \omega t} \frac{h^{2}-z^{2}}{h^{3}} \\
& =-\frac{3}{4} r \frac{h}{h}\left(1-\frac{z^{2}}{h^{2}}\right)
\end{aligned}
$$

and

$$
\begin{aligned}
v & =\frac{3}{2} j \omega \varepsilon h_{0} e^{j \omega t} \frac{z}{h}\left(1-\frac{z^{2}}{3 h^{2}}\right) \\
& =\frac{3}{2} h \frac{z}{h}\left(1-\frac{z^{2}}{h^{2}}\right),
\end{aligned}
$$

which is simply the Newtonian velocity field. Strictly speaking, all the $h$ 's in (18) and (19) should be replaced by $h_{0}$, since we have thrown out terms of order $O\left(\varepsilon^{2}\right)$ at the outset. However, we continue to write in the manner indicated in (18) and (19) for ease of comparison with the corresponding Newtonian solution.

Knowing the velocity field, the pressure field can be calculated, and hence the squeezing force is given by

$$
\begin{aligned}
F & =-\left.\pi \int_{0}^{a} r^{2}\left(\frac{\partial P}{\partial r}-\frac{\partial}{\partial r} \tau_{z z}\right)\right|_{z=h} d r \\
& =-\frac{3}{8} \pi a^{4} \eta^{*} \frac{h}{h}
\end{aligned}
$$

Since almost all polymeric liquids are shear-thinning in oscillatory shear flow, this study agrees with Tanner's remark [6] that, given the same squeezing rate, the squeezing force for a viscoelastic liquid tends to be smaller initially than that of a Newtonian liquid having the same zero-shear-rate viscosity. Furthermore, inertial effects do not seem to influence the result (20) and can be understood in the light of creeping flow near the surface of the plate where the integral over the pressure is performed.

However, away from the plate surface, the pressure field is influenced by inertia and can be shown to be

$$
P=-\frac{3}{4} \eta^{*}\left(a^{2}-r^{2}\right)\left[\frac{h}{h_{0}^{3}}+\frac{\rho}{2 \eta^{*}} \frac{\grave{h}}{h_{0}}\left(1-\frac{z^{2}}{h_{0}^{2}}\right)\right]+O\left(\varepsilon^{2}, h_{0}^{4} / a^{4}\right),
$$

where the pressure at the rim, $r=a$, is put to zero as a boundary condition. 
Equation (20) shows that dynamic properties of polymeric liquids can be measured on the plastometer in oscillatory mode. This may be useful to food and other technologists who have to deal with awkward, highly viscous, nonNewtonian pastes which suffer structural breakdown when subject to steady shearing.

\section{References}

[1] G. Brindley, J. M. Davies and K. Walters, "Elastico-viscous squeeze films. Part 1; Part 2: Superimposed rotation; Part 3: The torsional-balance rheometer", J. Non-Newt. Fluid Mech. 1 (1976), 19-37, 259-275, 277-286.

[2] R. R. Huilgol, Continuum mechanics of viscoelastic liquids (Hindustan Publ. Corp., Delhi, and Halsted Press, New York, 1975).

[3] J. M. Kramer, "Large deformations of viscoelastic squeeze films", Appl. Sci. Res. 30 (1974), 1-16.

[4] A. B. Metzner, "Extensional primary field approximations for viscoelastic media", Rheol. Acta 10 (1971), 434-444.

[5] P. Parlato, MSc Thesis, Univ. Delaware (1969).

[6] R. I. Tanner, "Some illustrative problems in the flow of viscoelastic non-Newtonian lubricants", Amer. Soc. Lub. Eng. Trans. 8 (1965), 179-183.

[7] G. Williams and R. I. Tanner, "Effects of combined shearing and stretching in viscoelastic lubrication", J. Lub. Tech. 92 (1970), 216-219.

Department of Mechanical Engineering

University of Newcastle

Newcastle

New South Wales 2308 